\title{
On the vaporization rate and flame shape of non-spherical droplets
}

\author{
John Palmore Jr* \\ Mechanical Engineering Department \\ Virginia Tech \\ Blacksburg, Virginia, 24061
}

Motivated by the study of spray combustion, this work addresses the combustion of non-spherical droplets. The combustion of spray is usually understood through the theory of droplet combustion, and improving this latter theory is the narrow aim of this work. The current work uses perturbation theory to derive a novel model for the vaporization of non-spherical droplets. Compared to previous efforts in this area, the work uses a physics-based approach by incorporating ideas from the asymptotic analysis of Taylor and Acrivos (1964, "On the deformation and drag of a falling viscous drop at low Reynolds number," Journal of Fluid Mechanics 18 (3), pp. 466-476). The perturbation strategy expands the droplet shape using spherical harmonics, and the theory characterizes the shape of the droplet via the Weber number. The introduction of this parameter is key as it is a parameter that can be easily measured in experiments, and thus it can be used to connect the theoretical results with application. The perturbation analysis is performed based around the classical solution of spherical droplet combustion in quiescent flow. The theory indicates that the effect of droplet deformation can be accounted for by a correction to the droplet combustion rate that is a simple polynomial function of the droplet Weber number. Results are compared to existing literature, and it confirms the established trend that deformed droplets vaporize faster than spherical droplets. Analysis of the flame shape reveals that the flame remains nearly spherical, however, the mean flame standoff changes with droplet shape. The extension of the theory to high Reynolds number conditions is briefly discussed.

\section{Important Nomenclature \\ $A_{f}$ Surface area of flame \\ $H$ Mean curvature of interface \\ $K$ Gaussian curvature of interface \\ $N$ Un-scaled interface normal vector \\ $P_{\ell} \quad \ell$-th order Legendre Polynomial \\ Re Reynolds number}

${ }^{*}$ Corresponding Author: palmore@vt.edu. ASME Member.

\author{
We Weber number \\ Z Zeldovich superscalar \\ $n$ Interface unit normal vector \\ $\dot{m}$ (Total) vaporization rate \\ $\dot{m}^{\prime \prime} \quad$ Vaporization rate per unit area. \\ $r_{f} \quad$ Flame standoff \\ $r$ Radial coordinate in spherical coordinates \\ $\varphi$ Azimuthal angle in spherical coordinates \\ $\theta$ Polar angle in spherical coordinates \\ $\varepsilon$ Perturbation parameter \\ $\Phi$ Velocity potential \\ $\eta \quad$ Interface shape function
}

\section{Introduction}

This work addresses the vaporization and combustion of nonspherical liquid droplets as it is a topic relevant to spray combustion. In spray combustion, the liquid fuel is injected into the combustor where it atomizes into droplets, the droplets vaporize, and the vapor undergoes combustion. This paper focuses on the vaporization and combustion of an isolated liquid droplet and studies the effects of droplet nonsphericity through perturbation analysis.

As noted in the review by [1], in real flows droplets deform away from spherical shapes due to a balance between aerodynamic and surface tension forces. Quantifying this effect is important for comparing real spray droplets to experimental studies. Experiments often focus on characterizing the combustion of relatively large droplets with sizes on the order of $1 \mathrm{~mm}$, whereas the droplets seen in actual spray are often on the order of $10 \mu \mathrm{m}$ or less. Many phenomena behave differently for such large droplets. It is known, for example, that soot formation and radiative flame extinction behave fundamentally differently for large droplets [2,3,4]. An analogous 
issue comes in matching the vaporization and flame properties between experiment, theory, and practice. A smaller droplet with the same Reynolds number must necessary experience a faster velocity, and therefore it sees greater deformation from aerodynamic forces. In a fuel spray evolving through a turbulent gas flow, this effect will be unimportant for the smallest spray droplets. However, the largest droplets are often on the order of the Kolmogorov length [5], and accordingly can experience significant deformation due to Kolmogorov-scale turbulent flow disturbances. That inspires a study on the effect deformation on vaporization which will be the focus of this study. Importantly, this work will demonstrate a simple strategy for relating the vaporization of actual, deforming droplets with the theory of spherical droplets via the Weber number.

The isolated droplet configuration was chosen because it has become a canonical test case of droplet combustion, and often serves as the basis for models of spray. The combustion of isolated droplets may be directly relevant in certain cases such as in extremely dilute spray, or during the process of flame kernel formation and ignition [6]. However in practice droplets are close enough to each other to cause interaction effects. Due to this interaction, it is known that the nature of combustion for a closely-spaced droplets is different than that of isolated droplets [7, 8,9]. Accordingly, these effects must be addressed in future efforts. However, by first addressing the isolated droplet, this work connects with standard practice in the field.

While the vaporization and combustion of droplets has been extensively studied theoretically, experimentally, and numerically [10, 11, 12, 13, 14, 15, 16, 17, 18, 19], there is still no detailed theory for deformed droplets [20]. In order to make analysis of the problem more tractable, a number of simplifying assumptions are typically made to the problem. Common assumptions for theoretical studies are that the liquid is pre-heated to its saturation temperature, the liquid density is much greater than the gas density, and the liquid viscosity is much greater than the gas viscosity. These assumptions ensure that there is negligible heat and momentum transport in the liquid phase, and accordingly the analysis of these phenomena is neglected. Therefore, the droplet combustion is usually modeled as a gas phase heat and mass transfer process. Modeling approaches have extended usable knowledge to convective flow [21, 16] and non-equilibrium thermodynamic effects [22], however, all common models still rely on the spherical droplet assumption.

One manner of studying droplet vaporization and combustion without limiting assumptions is through direct numerical simulations. The application of this strategy to flows in three dimensions has become a popular topic of research, and now involves many studies [23, 24, 25, 26, 27, 28, 29]. However, in order for information from such studies to be transferable to practical knowledge for experiments, a large body of these simulations must be performed. Currently, it is too computationally expensive to achieve this task. Accordingly, a strategy that involves a theoretical approach is required.
Grow [30] performed a theoretical study on the quasi-steady heat and mass transfer from ellipsoidal coal particles by solving Laplace's equation in ellipsoidal coordinates. The work demonstrated that the combustion rate of such particles was only slightly higher than of spherical coal particles. However, [30] analysis was performed for diffusional heat transfer, so it did not consider the Stefan flow effects associated to vaporization. Tonini, Cossali, and colleagues use a similar strategy to study vaporization of ellipsoidal droplets [31, 32, 33]. In this case they demonstrated that the vaporization rate of the droplets was enhanced by the deformation of the droplets.

While it is known that droplets deform to ellipsoids in the first approximation, it is also known that higher approximations are non-ellipsoidal [34]. Therefore many of the interface shapes implied by [31], particularly for large deviations from spherical shape, are not physically realizable. This makes comparison to experiments challenging.

Mashayek [35] studied the problem computationally considering vaporization, however, the author was able to include more complex droplet shapes. This was performed by studying the problem using spherical coordinates, and by defining the interface shape to be a function of the polar angle, $\theta$. (The problem assumes axisymmetry, so the azimuthal angle is unimportant.) The work performed simulations of droplets of varying shapes to characterize the effect of shape on vaporization. Consistent with the other literature, the work demonstrated an increase in vaporization rate for deformed droplets. [35] also demonstrated that the local variations of vaporization rate on the interface is related with the local interface curvature. However, [35] was interested in studying the vaporization of oscillating droplets. As a consequence, the work considered the vaporization of arbitrary shapes which occur during the oscillation process, and these shapes do not reflect the properties of steady-state vaporization of a deformed droplet.

\subsubsection{Specification of the Approach and its Limitations}

The approach taken in this paper is to study the vaporization and combustion of deformed droplets using a perturbation expansion around the sphere solution. A strategy similar to [35] is used to represent the shape of the interface, however, the actual shapes used will come from a physicsbased understanding of the problem [34]. Other than the assumption of non-sphericity, the analysis caries over many of the assumptions made in the classical solution [36]. It is assumed that heat and momentum transport in the liquid are negligible. The gas phase is assumed to undergo quasisteady vaporization and combustion with constant fluid properties. It is further assumed that the fuel consists of only a single component, that the Lewis number is unity, and that combustion occurs in a negligibly thin flame sheet. Finally, as is the case for the classical solution, the droplet vaporizes and combusts in a quiescent environment, $i$. $e$. zero ambient velocity.

This appears to lead to a logical inconsistency because the 
presence of non-zero convective flow is the physical mechanism that causes droplets to demonstrate non-spherical shapes. However, this inconsistency exists in appearance only. It can be resolved by an interpretation of the problem as relating to an asymptotic analysis approach. The shape of the droplet is controlled by the balance between aerodynamic pressure and surface tension, and this balance is dominated by the flow from the outer freestream region. Its expression is given by the solution from [34]. For this interpretation to be correct, it is required that the non-uniformity in the momentum flux due to vaporization must be weak compared to the freestream convective flow. With these assumptions, the aerodynamic stresses associated to vaporization are negligible in comparison to the freestream stresses, and they have negligible effect on the droplet shape.

One further assumption was necessary to make the problem tractable: it is assumed that the Stefan flow velocity is irrotational. The irrotational assumption is necessary to define a unique velocity field from knowledge of only the vaporization rate and interface boundary conditions. These assumptions are consistent with what was used in previous analytical studies such as [31]. These overall set of assumptions implies a limited band of applicability for the current work; however, despite these limitations, it still makes a step forward in the state of the art for analytical theory of droplet vaporization. The paper is organized as such Section 2 details the methodology used for the theoretical study. Section 3 demonstrates the solution of the equations. Section 4 applies the methods to the given problem and discusses the implications thereof including the effect of droplet non-sphericity on the vaporization rate, flame shape, and flame standoff. Direct comparisons are made to the results from [31,35]. Section 5 concludes the work.

\section{Methodology}

The paper considers the quasi-steady vaporization of a deformed droplet, and solves this problem as a perturbation of the spherical droplet solution. The problem is solved in spherical coordinates following the convention of Batchelor [37] with radius $r$, polar angle $\theta \in[0, \pi]$, and azimuthal angle $\varphi \in[0,2 \pi]$. Denote the unit vectors in the three directions as $\boldsymbol{e}_{r}, \boldsymbol{e}_{\theta}$, and $\boldsymbol{e}_{\varphi}$, respectively. The interface of the droplets is characterized by a vector $\boldsymbol{r}_{s}$, which represents the displacement from the origin to the interface as a function of $\theta$ and $\varphi$. Using $\boldsymbol{r}_{s}$ all relevant geometric information about the surface may be computed.

In this work we will be interested in shapes that demonstrate symmetry in the azimuthal direction. This is the case most relevant to the combustion of liquid fuel droplets because droplets preferentially deform into structures with this symmetry. The review by [1] notes that mildly deformed droplets tend towards spheroidal axisymmetric shapes. This assumption has been used in previous theoretical and numerical studies of flow around both vaporizing [38, 35, 31] and non-vaporizing non-spherical droplets [34,39]. Accordingly the axisymmetry assumption will be used for this work.

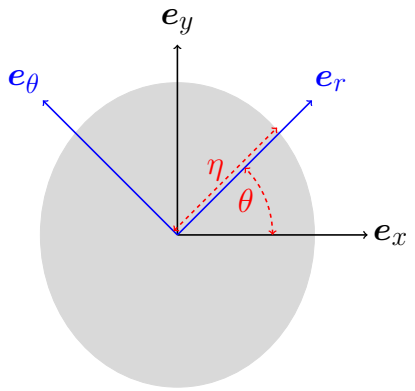

Fig. 1: Coordinate system. Due to axisymmetry, only a single plane of the 3D geometry need be shown.

Note, that this assumption implicitly limits the domain of applicability of the study to only droplets that experience mild deformations.

From this assumption, we can write $\boldsymbol{r}_{s}=\eta \boldsymbol{e}_{r}$ where $\eta$ is a function of only $\theta$. The coordinates are depicted in Fig. 1

Following [40], the interface tangent vectors are

$$
\begin{aligned}
\boldsymbol{t}_{\theta} & =\eta \boldsymbol{e}_{\theta}+\partial_{\theta} \eta \boldsymbol{e}_{r} \\
\boldsymbol{t}_{\varphi} & =\eta \boldsymbol{e}_{\varphi}
\end{aligned}
$$

and the normal vector is

$$
\boldsymbol{n}=\frac{\boldsymbol{t}_{\theta} \times \boldsymbol{t}_{\varphi}}{\left|\boldsymbol{t}_{\theta} \times \boldsymbol{t}_{\varphi}\right|}
$$

A somewhat more convenient variable for manipulation is the un-scaled normal vector,

$$
\boldsymbol{N}=\boldsymbol{t}_{\theta} \times \boldsymbol{t}_{\varphi}=\eta^{2} \sin \theta \boldsymbol{e}_{r}-\eta \partial_{\theta} \eta \sin \theta \boldsymbol{e}_{\theta}
$$

The governing equations are expressed in non-dimensional form. The dimensional quantities used as references are the droplet radius $\left(R_{0}\right)$, gas density $\left(\rho_{G}\right)$, gas specific heat $\left(c_{p}\right)$, and species diffusivity $(D)$. Consistent with the unity Lewis number assumption, all diffusivities are equal. The coordinates and field variables are non-dimensionalized for this analysis using $R_{0}$ as the reference length, $R_{0}^{2} / D$ as the reference time, $\rho_{G} R_{0}^{3}$ as the reference mass, and $\left(D / R_{0}\right)^{2} / c_{p}$ as the reference temperature. Since the solution assumes axisymmetry, all field variables are a function of only $r$ and $\theta$. For brevity, $\nabla$ notation is used to represent vector differentiation. The operators are defined as follows.

The gradient of a scalar quantity $\Xi$ is then represented as

$$
\nabla \Xi=\boldsymbol{e}_{r} \partial_{r} \Xi+\frac{\boldsymbol{e}_{\theta}}{r} \partial_{\theta} \Xi
$$

and the divergence of a vector $\boldsymbol{\Xi}=\boldsymbol{e}_{r} \boldsymbol{\Xi}_{r}+\boldsymbol{e}_{\theta} \Xi_{\theta}$ is

$$
\nabla \cdot \boldsymbol{\Xi}=\frac{1}{r^{2}} \partial_{r}\left(r^{2} \Xi_{r}\right)+\frac{1}{r \sin \theta} \partial_{\theta}\left(\sin \theta \Xi_{\theta}\right)
$$


The Laplacian of a scalar is equivalent to the divergence of the gradient, and is represented

$$
\nabla^{2} \Xi=\frac{1}{r^{2}} \partial_{r}\left(r^{2} \partial_{r} \Xi\right)+\frac{1}{r^{2} \sin \theta} \partial_{\theta}\left(\sin \theta \partial_{\theta} \Xi\right)
$$

We consider the governing equations of a non-spherical droplet vaporizing with no external flow. The governing equations are the expression for advection and diffusion of the species mass fractions and temperature. For an irrotational flow, the velocity can be expressed as the gradient of a potential, $\boldsymbol{u}=\nabla \Phi$. Accordingly, for a flow that is both incompressible and irrotational

$$
\nabla^{2} \Phi=0
$$

It is assumed that the flow is oriented outwards from the surface of the droplet, or equivalently that the projection of the velocity onto the surface is null. This implies

$$
\begin{array}{r}
\boldsymbol{t}_{\theta} \cdot \nabla \Phi=0 \\
\partial_{\theta} \Phi+\partial_{\theta} \eta \partial_{r} \Phi=0
\end{array}
$$

The gas phase is described by its chemical composition and temperature. Denote the mass fractions of fuel and oxidizer as $Y_{F}$ and $Y_{O}$ respectively, and the temperature as $T$. They satisfy [41]

$$
\begin{aligned}
\nabla \cdot\left(Y_{F} \nabla \Phi\right)-\nabla^{2} Y_{F} & =\dot{\omega} \\
\nabla \cdot\left(Y_{O} \nabla \Phi\right)-\nabla^{2} Y_{O} & =\frac{\dot{\omega}}{v} \\
\nabla \cdot(T \nabla \Phi)-\nabla^{2} T & =-Q \dot{\omega}
\end{aligned}
$$

where $\omega$ is the reaction rate, $v$ is the stoichiometric fuel air ratio and $Q$ is the heat release from combustion. The equations have been non-dimensionalized from their original form using the established reference conditions. It is assumed values for $Y_{F}, Y_{O}$, and $T$ are known on the interface, ${ }_{s}$, and at the far field, $\infty$. At the interface, conservation of species mass and energy require

$$
\begin{aligned}
\left(Y_{F, s}-1\right) \boldsymbol{n} \cdot \nabla \Phi & =\boldsymbol{n} \cdot \nabla Y_{F} \\
\left(Y_{O, s}\right) \boldsymbol{n} \cdot \nabla \Phi & =\boldsymbol{n} \cdot \nabla Y_{O} \\
H_{v} \boldsymbol{n} \cdot \nabla \Phi & =\boldsymbol{n} \cdot \nabla T
\end{aligned}
$$

In solving these equations it is convenient to introduce Zeldovich variables,

$$
\begin{aligned}
& Z^{F}=Y_{F}-v Y_{O} \\
& Z^{T}=\left(T-T_{S}\right)+v Q Y_{O}
\end{aligned}
$$

which both satisfy

$$
\nabla \cdot(Z \nabla \Phi)-\nabla^{2} Z=0
$$

where $Z$ represents either $Z^{F}$ or $Z^{T}$. The boundary conditions are

$$
\begin{aligned}
\left(Z_{s}^{F}-1\right) \boldsymbol{n} \cdot \nabla \Phi & =\boldsymbol{n} \cdot \nabla Z^{F} \\
Z^{T} \boldsymbol{n} \cdot \nabla \Phi & =\boldsymbol{n} \cdot \nabla Z^{T}
\end{aligned}
$$

or briefly as

$$
g(Z) \boldsymbol{n} \cdot \nabla \Phi=\boldsymbol{n} \cdot \nabla Z
$$

which can represent either of the previous equations.

\subsection{Perturbation Strategy}

The work considers a regular perturbation analysis using a arbitrary small parameter, $\varepsilon$. (It will later be shown that droplet's Weber number is a physically meaningful choice for $\varepsilon$.) We expand each field variables in the form,

$$
\begin{aligned}
& \eta=\eta_{0}+\varepsilon \eta_{1}+\varepsilon^{2} \eta_{2} \\
& Z=Z_{0}+\varepsilon Z_{1}+\varepsilon^{2} Z_{2} \\
& \Phi=\Phi_{0}+\varepsilon \Phi_{1}+\varepsilon^{2} \Phi_{2}
\end{aligned}
$$

and so on. It can be shown that when the droplet is not too greatly deformed from spherical shape, $\eta_{0}=1$. As is typical for these problems, these expression will be substituted into the governing equations and grouped by powers of $\varepsilon$ to determine the perturbed equations. Terms of order $\varepsilon^{3}$ and higher are ignored. The resulting equations are

To $\varepsilon^{0}$

$$
\begin{aligned}
\nabla^{2} \Phi_{0} & =0 \\
\nabla \cdot\left(Z_{0} \nabla \Phi_{0}\right)-\nabla^{2} Z_{0} & =0
\end{aligned}
$$

To $\varepsilon^{1}$

$$
\begin{aligned}
\nabla^{2} \Phi_{1} & =0 \\
\nabla \cdot\left(Z_{1} \nabla \Phi_{0}\right)-\nabla^{2} Z_{1} & =-\nabla \cdot\left(Z_{0} \nabla \Phi_{1}\right)
\end{aligned}
$$

To $\varepsilon^{2}$

$$
\begin{aligned}
\nabla^{2} \Phi_{2} & =0 \\
\nabla \cdot\left(Z_{2} \nabla \Phi_{0}\right)-\nabla^{2} Z_{2} & =-\nabla \cdot\left(Z_{0} \nabla \Phi_{2}\right)-\nabla \cdot\left(Z_{1} \nabla \Phi_{1}\right)
\end{aligned}
$$


The representation of the boundary conditions is more challenging. The boundary conditions are to be evaluated at $\eta(\theta)$. However, this is challenging to resolve in practice because the interface shape is also part of the perturbation analysis. Since $\eta=1+O(\varepsilon)$, the boundary condition can be moved to $r=1$ via formal application of the Taylor series [42], for example

$$
\left.\Phi\right|_{r=\eta}=\Phi+\varepsilon \eta_{1} \partial_{r} \Phi+\varepsilon^{2}\left(\eta_{2} \partial_{r} \Phi+\frac{\eta_{1}^{2}}{2}\left(\partial_{r}\right)^{2} \Phi\right)
$$

where the terms on the right side of the equation are understood to be evaluated at $r=1$.

Using this strategy, the resulting boundary conditions for $\Phi$ can be rewritten at $r=1$ as

$$
\begin{aligned}
& \varepsilon^{0}: \partial_{\theta} \Phi_{0}=0 \\
& \varepsilon^{1}: \partial_{\theta} \Phi_{1}=-\partial_{\theta} \eta_{1} \partial_{r} \Phi_{0} \\
& \varepsilon^{2}: \partial_{\theta} \Phi_{2}=-\partial_{\theta} \eta_{1} \partial_{r} \Phi_{1}-\partial_{\theta} \eta_{2} \partial_{r} \Phi_{0}
\end{aligned}
$$

To recover the solution in this form the result $\varepsilon^{0}$ is substituted into the relation for $\varepsilon^{1}$, and these results are subsequently used in the relation $\varepsilon^{2}$. The superscalar boundary conditions at infinity imply

$$
\begin{aligned}
& Z_{0}(r \rightarrow \infty)=Z_{0, \infty} \\
& Z_{1}(r \rightarrow \infty)=0 \\
& Z_{2}(r \rightarrow \infty)=0
\end{aligned}
$$

and the interface conditions can be rewritten at $r=1$ as

$$
\begin{aligned}
\varepsilon^{0}: g \partial_{r} \Phi_{0} & =\partial_{r} Z_{0} \\
\varepsilon^{1}: g\left[\eta_{1} \partial_{r}^{2} \Phi_{0}+\partial_{r} \Phi_{1}\right] & =\eta_{1} \partial_{r}^{2} Z_{0}+\partial_{r} Z_{1}
\end{aligned}
$$

and for $\varepsilon^{2}$,

$$
\begin{array}{r}
g\left[\eta_{2} \partial_{r}^{2} \Phi_{0}+\frac{\eta_{1}^{2}}{2} \partial_{r}^{3} \Phi_{0}+\eta_{1} \partial_{r}^{2} \Phi_{1}+\partial_{r} \Phi_{2}+\left(\partial_{\theta} \eta_{1}\right)^{2} \partial_{r} \Phi_{0}\right] \\
=\left[\eta_{2} \partial_{r}^{2} Z_{0}+\frac{\eta_{1}^{2}}{2} \partial_{r}^{3} Z_{0}+\eta_{1} \partial_{r}^{2} Z_{1}+\partial_{r} Z_{2}-\partial_{\theta} \eta_{1} \partial_{\theta} Z_{1}\right]
\end{array}
$$

where $g$ was defined previously in Eq. 22,

\subsection{Physical Modeling Strategy}

The crux of this work is to rely on a physics-based representation of the interface shape rather than imposing an arbitrary shape. Taylor and Acrivos [34] studied analytically the deformation of a falling droplet. They used a matched asymptotic perturbation strategy which built the solution for the droplet shape in the low Reynolds number limit. They demonstrated agreement with established experimental shapes for droplets and bubbles. Their work identified the key parameters that govern the droplet shape as the Reynolds and Weber numbers,

$$
\begin{aligned}
R e & =\frac{\rho_{G} U d}{\mu_{G}} \\
W e & =\frac{\rho_{G} U^{2} d}{\sigma}
\end{aligned}
$$

where $\rho_{G}$ and $\mu_{G}$ represent the gas density and viscosity, $d=$ $2 R_{0}$ is the diameter, $\sigma$ is the surface tension between phases, and $U$ is the freestream velocity. The solution therein derived is rather complex, however it can be greatly simplified in the case where the liquid viscosity is assumed to be much greater than that of the gas. In that limit the solution becomes,

$$
\eta_{T A}=1-\frac{81}{640} W e P_{2}(\cos \theta)-\frac{2673}{44800} \frac{W e^{2}}{R e} P_{3}(\cos \theta)
$$

Note that [34] defined $R e$ and $W e$ using $R_{0}$ in place of $d$, so the values demonstrated here are slightly different than their values.

Here $P_{\ell}$ is the Legendre polynomial [43]. The polynomials satisfy

$$
P_{\ell}(x)=\frac{1}{2^{\ell} \ell !}\left(\partial_{x}\right)^{l}\left(1-x^{2}\right)^{\ell}
$$

with the orthogonality conditions

$$
\int_{0}^{\pi} P_{\ell}(\cos \theta) P_{k}(\cos \theta) \sin \theta d \theta=\frac{2}{2 \ell+1} \delta_{\ell k}
$$

with $\delta_{\ell k}$ as the Kronecker delta. The Legendre polynomials are related to the so-called spherical harmonics [43], and therefore are ubiquitous to analytical solutions in spherical coordinates.

Equation (44) suggests the use of $\varepsilon=W e$ for the perturbation parameter. However, this expression introduces the Reynolds number as an additional parameter, and therefore a bivariate analysis using We and Re may be required. However, for this study, we only consider how variations of We affect vaporization at fixed $R e$. Accordingly, a single variable $W e$ is used to parameterize the perturbation study. To generalize the discussion slightly, we consider the case where the expansion $\eta$ satisfies,

$$
\eta=1+\varepsilon h_{1,2} P_{2}+\varepsilon^{2}\left(h_{2,0}+h_{2,3} P_{3}\right)
$$


and Eq. (44) is recovered for

$$
\begin{aligned}
& h_{1,2}=-\frac{81}{640} \\
& h_{2,3}=-\frac{2673}{44800 R e}
\end{aligned}
$$

It can be demonstrated that the additional term, $h_{2,0}$, must be introduced to ensure that the volume of the deformed droplet matches that of the spherical droplet to order $\varepsilon^{2}$. This term was not necessary in [34] because it is of order $R e^{4}$, and therefore is beyond the scope of their study which focused on $R e$ effects. However, this study focuses on $W e$, and therefore the term is required to preserve the solution to order $\varepsilon^{2}=W e^{2}$. To ensure volume conservation, the term must satisfy,

$$
h_{2,0}=-\frac{1}{5} h_{1,2}^{2}
$$

which yields that the term that would be consistent with [34] is

$$
h_{2,0}=-\frac{6561}{1024000}
$$

This generalization allows the consideration of both prolate and oblate shapes by varying $h_{1,2}$. In their analysis, [34] notes that in practice only oblate shapes occur in droplets, so it is expected to the be most important for the spray combustion application. However, introducing an option for prolate shape allows for comparison to that result in [31].

\section{Solution}

\subsubsection{Order $\varepsilon^{0}$}

Unsurprisingly, the governing equations and boundary conditions of the $\varepsilon^{0}$ solution are equal to the spherical problem, and the same result is recovered.

$$
\begin{aligned}
\Phi_{0} & =f_{0}\left(\frac{1}{r}-1\right) \\
Z_{0} & =\left.Z_{0}\right|_{s}+g\left\{\exp \left[f_{0}\left(\frac{1}{r}-1\right)\right]-1\right\}
\end{aligned}
$$

The constant $f_{0}$ is to be solved from the previous equations. Evaluating the Equation 39 at $r=1$, the $\varepsilon^{0}$ order solution yields

$$
f_{0}=-\log \left(1+\frac{\left.Z_{0}\right|_{\infty}-\left.Z_{0}\right|_{s}}{g}\right)
$$

It can be demonstrated that the total vaporization rate (to $\varepsilon^{0}$ ) is

$$
\begin{aligned}
\dot{m} & =2 \pi \int_{0}^{\pi} \partial_{r} \Phi_{0} \sin \theta d \theta \\
& =-4 \pi f_{0} \\
& =4 \pi \log \left(1+\frac{\left.Z_{0}\right|_{\infty}-\left.Z_{0}\right|_{s}}{g}\right)
\end{aligned}
$$

Substituting the value

$$
B=\frac{\left.Z_{0}\right|_{\infty}-\left.Z_{0}\right|_{s}}{\left.Z_{0}\right|_{s}-1}
$$

and re-expressing dimensionally yields

$$
\dot{m}=4 \pi \rho_{G} D R_{0} \log (1+B)
$$

which agrees with the classical result.

\subsubsection{Order $\varepsilon^{1}$}

The potential satisfies Laplace's equation to all orders. Laplace's equation is a well-studied problem, and the solution that is bounded at infinity can be shown to be

$$
\Phi_{1}=\sum_{l=0}^{\infty} \frac{f_{1, \ell}}{r^{\ell+1}} P_{\ell}(\cos \theta)
$$

where $f_{1, \ell}$ are constants. Using this solution, the coefficients can be solved from the Eq. (34). The boundary condition, Eq. (34) becomes

$$
\sum_{\ell=0}^{\infty}\left[f_{1, \ell}-f_{0} h_{1, \ell}\right] \partial_{\theta} P_{\ell}=0
$$

which implies $f_{1, \ell}=h_{1, \ell} f_{0}$ for $\ell>0$. This equation does not provide a solution for $\ell=0$ since $\partial_{\theta} P_{0}=0$. To generate one, we assume that the perturbation decays rapidly at infinity, i.e.

$$
\lim _{r \rightarrow \infty} \frac{\Phi_{1}}{\Phi_{0}}=0
$$

This is mathematically equivalent to assuming that far field boundary condition on velocity is captured solely by the $\varepsilon^{0}$ solution, and this yields $f_{1,0}=0$.

The form of the previous equations suggests a solution using separation of variables as

$$
Z_{1}=\sum_{l=0}^{\infty} \zeta_{1, \ell} P_{\ell}(\cos \theta)
$$


where the $\zeta_{1, \ell}$ are functions of $r$ rather than being constants. Substitution of this into the governing partial differential equation yields an ordinary differential equation for the $\zeta_{1, \ell}$,

$$
\partial_{r}\left(r^{2} \partial_{r} \zeta_{1, \ell}\right)+f_{0} \partial_{r} \zeta_{1, \ell}-\ell(\ell+1) \zeta_{1, \ell}=-\partial_{r} Z_{0}(\ell+1) f_{1, \ell} r^{-\ell}
$$

A sketch of the solution process is demonstrated in the Supplemental Material. The most relevant result of the analysis is the fact that the function $\zeta_{1, \ell}$ is non-zero only when the corresponding shape perturbation parameter $h_{1, \ell}$ is non-zero.

\subsubsection{Order $\varepsilon^{2}$}

The solution to order $\varepsilon^{2}$ proceeds analogously to that of order $\varepsilon^{1}$. The field variables are expanded as

$$
\begin{aligned}
& \Phi_{2}=\sum_{l=0}^{\infty} \frac{f_{2, \ell}}{r^{\ell+1}} P_{\ell}(\cos \theta) \\
& Z_{2}=\sum_{l=0}^{\infty} \zeta_{2, \ell} P_{\ell}(\cos \theta)
\end{aligned}
$$

and the coefficients are found from the boundary conditions. The boundary conditions Eqs. (35) and (41) are more challenging to deal with a priori due to the nonlinearity of the equations. Accordingly, this section restricts attention to the specific case of an equation of the form Eq. (47). The solution sketch is given in the Supplementary Material.

The resulting expressions for $\Phi_{2}$ are

$$
\begin{aligned}
f_{2,0} & =0 \\
f_{2,2} & =\frac{4}{7} h_{1,2}^{2} f_{0,0} \\
f_{2,3} & =h_{2,3} f_{0,0} \\
f_{2,4} & =\frac{36}{35} h_{1,2}^{2} f_{0,0}
\end{aligned}
$$

and all other coefficients $f_{2, \ell}$ are null. Unlike the solutions at first order, the second order solutions $\Phi_{2}, Z_{2}$ are not in phase with the second order perturbation $\eta_{2}$. This follows from the non-linearity of the boundary conditions, which introduces information at frequencies both higher and lower than those contained in the perturbation, $\eta$. Since the pattern of oscillation of these functions is different, different strategies much be used to solve each function.

Simplified forms for the differential equations for the $\zeta_{2, \ell}$ functions are given in the Supplemental Material. Explicit expression are not written here for the solutions. This is not a limitation, as the primary theoretical target for this work is computation of the vaporization rate, which can be computed using the $\Phi$ function alone. That function has been written explicitly to order $\varepsilon^{2}$. It will also be demonstrated in Section 4.4 that the analytical expression for $\zeta_{2, \ell}$ are not required to compute the mean flame location to the appropriate order, so this phenomena can also be investigated.

\section{Results and Discussion}

The work has demonstrated the perturbation theory and the solutions of the equations. Using the expression for $\eta$ (Eq. (47)) closed-form expressions for the velocity and scalar fields can be generated.

This section demonstrates several analyses on how to gain practical information such as the vaporization rate and flame shape from knowledge of $\eta$. And discusses these trends generally in comparison to the base $(\varepsilon=0)$ solution and previous analyses of droplet vaporization such as [35, 31].

\subsection{The Vaporization Rate}

One of the most important quantities to note for droplet vaporization and combustion is the vaporization rate. Given the symmetries of this problem, the expression can be demonstrated to satisfy,

$$
\dot{m}=2 \pi \int N \cdot \nabla \Phi \sin \theta d \theta
$$

A somewhat surprising result that can be verified after the fact, is that the information given above in the solution of or$\operatorname{der} \varepsilon^{2}$ is actually sufficient to compute the vaporization rate to order $\varepsilon^{3}$. To do this we introduce, $\eta_{3}$ and $\Phi_{3}$ as unknown functions and expand them in a manner similar to what was done for $\eta_{1}, \eta_{2}$ and $\Phi_{1}, \Phi_{2}$. Due to a combination of the surface projection law Eq. (10) and the orthogonality properties of the Legendre functions, none of the $\eta_{3}, \Phi_{3}$ functions survive the integration.

On expanding these expressions and taking the integration, the result becomes

$$
\dot{m}=-4 \pi f_{0,0}\left[1+\varepsilon^{2} \frac{54}{5} h_{1,2}^{2}+\varepsilon^{3}\left(\frac{6}{5} h_{1,2}^{2}+\frac{158}{35} h_{1,2}^{3}+\frac{57}{35} h_{1,2}^{4}\right)\right]
$$

It is understood that Eq. (72) is non-dimensional, and the dimensional expression is found by scaling by $\rho_{G} D R_{0}$. In Eq. (72), it is interesting to note that the term $h_{23}$ does not appear in this expression, and therefore there is no direct Reynolds number dependence of this result. This appears to contradict theory and established literature on droplets [41], however this result is not entirely surprising. The primary influence of Reynolds number is on boundary layer development. Since this solution does not consider boundary layer flow over the droplet, it does not recover this phenomenon. Accordingly, the theory is only directly applicable in the limit of very small Reynolds number. Although the solution does not capture boundary layer growth or other Reynolds number effects, it is still useful for analysis and comparison to the established literature on vaporization and combustion of non-spherical droplets.

This result is compared to the result of [31] who consider the solution of an ellipsoidal particle. Their work writes the vaporization rate using the aspect ratio $\beta$ defined such that 
$\beta<1(\beta>1)$ for oblate (prolate) spheroids. Expanding this in ellipsoidal coordinates they recovered,

$$
\dot{m}=-4 \pi f_{0,0} \frac{\sqrt{\left|1-\beta^{2}\right|}}{\sqrt[3]{\beta}} \begin{cases}\frac{1}{\pi-2 \arctan e^{\xi_{0}}} & \text { oblate } \\ \frac{1}{\log \frac{\xi_{0}+1}{e_{0}-1}} & \text { prolate }\end{cases}
$$

where $\xi_{0}$ is the value of the ellipsoidal coordinate variable $\xi$ that defines the droplet surface.

It is difficult to directly analyze this expression. The natural comparisons for the solution would be to the sphere solution, however the ellipsoidal coordinates demonstrate a singularity in the spherical $(\beta=1)$ limit. For example, naive substitution of $\beta=1$ into the expression yields to an expression of form $0 / 0$. Hence the solution is non-smooth there, and in fact Eq. (73) demonstrates branching at that point. This makes the equation challenging for inferences about the behavior of droplet at small deformations. However, it is also established that at large deformation, droplets begin to deviate from ellipsoids shape [34], so the [31] theory loses validity in this limit as well. Even still, it is interesting to compare the results of Eq. (73) with Eq. (72).

The vaporization rate is an integral quantity, so it does not give information about local variations in the velocity or scalar fields. Accordingly, another quantity of interest is the vaporization rate per unit area,

$$
\dot{m}^{\prime \prime}=\boldsymbol{n} \cdot \nabla \Phi
$$

which is evaluated locally at each location on the interface, and therefore is a function of $\theta$. This quantity can also be used to define a local Stefan flow velocity, $\dot{m}^{\prime \prime} / \rho_{G}$. A corresponding Stefan flow Weber number can be constructed using the maximum value as

$$
W e_{S}=\frac{\left(\dot{m}^{\prime \prime}\right)_{\mathrm{Max}}^{2} d}{\rho_{G} \sigma}
$$

Sphere Limit Taking the two limits in Eq. (73) separately as $\beta \rightarrow 1$ reveals that the sphere solution is recovered. In Eq. (72) it is simple to set $\varepsilon=0$ and recover the same result.

Nullity of first variation The total vaporization rate $(\dot{m})$ only varies to second order in $\varepsilon$, that is the first variation of this quantity is null. This trend is not as readily apparent in Eq. (73). However, by differentiating and taking the limits separately of the two expressions in Eq. (73), the trend also is revealed. The nullity of the first variation is somewhat surprising since from Section 3 it is can be demonstrated that the local vaporization rate $\left(\dot{m}^{\prime \prime}\right)$ varies to first variation.

Positive definite second variation From Eq. (72), it is clear that the second variation is positive definite since it is written in terms of $h_{1,2}^{2}$. When combined with the fact that first variation is null, this suggests that small deformations always increase the vaporization rate. This trend cannot be found analytically by limits in the [31] solution because a cusp occurs in their solution. However, plotting the solution as they have in their paper (their Figure 2) suggests a similar trend.

Prolate shapes vaporize faster than oblate shapes From Eq. (72), we can also analyze the third variation. It is again clear that when $h_{1,2}$ is positive (i.e. prolate shape) the third variation is positive, so vaporization is always enhanced. However, when $h_{1,2}$ is negative (i.e. oblate shape), the positivity or negativity of the third variation depends on this quantity's actual magnitude. However, in either case this value will be less in absolute value than third variation where $h_{1,2}$ is positive. From this we can say that for the same $\left|h_{1,2}\right|$ the prolate shape will vaporize faster. That trend agrees with the result of [31] (see their Figure 2). This result also suggest that the cubic solution is the least order solution capable of distinguishing prolate and oblate shapes, which could have modeling implications in future studies.

\subsection{Area's Effect on Vaporization Rate}

It has now been demonstrated that the solution derived in this paper is consistent both with the established sphere theory, and it is consistent with the [31] theory in the small deformation limit. One commonly held belief in the community of droplet vaporization is that the vaporization of deformed droplets is only higher due to increased surface area. This section will falsify this belief. The expression given by [31] in Eq. (73) hints at this idea, since their expression depends only on aspect ratio, $\beta$, and not upon the area directly. However, the direct application of this idea to non-ellipsoidal droplets is still an open question. There is some ambiguity as to what it would mean for the vaporization enhancement to be caused by surface area increase. Accordingly, the idea is tested from two perspectives in this work.

\subsubsection{Area Scaling}

One interpretation of this idea is that the vaporization rate per unit area is the same for deformed and spherical droplets. However, because of the higher area of the deformed droplet the net vaporization is higher. Accordingly, we might expect that by multiplying the sphere solution by the area ratio, we would recover the solution form Eq. (72). The area of our deformed droplet can be computed as

$$
A=2 \pi \int_{0}^{\pi} \eta \sin \theta \sqrt{\eta^{2}+\left(\partial_{\theta} \eta\right)^{2}} d \theta
$$

which follows from [40]. Substitution of the relevant definition for $\eta$ yields

$$
A=4 \pi\left(1+\varepsilon^{2} \frac{2}{5} h_{1,2}^{2}-\varepsilon^{3} \frac{4}{105} h_{1,2}^{3}\right)
$$


Therefore the vaporization rate for the sphere can be scaled as,

$$
\begin{aligned}
\dot{m}_{\text {scale }} & =\frac{A}{A_{\text {sphere }}} \dot{m}_{\text {sphere }} \\
& =-4 \pi f_{0,0}\left(1+\varepsilon^{2} \frac{2}{5} h_{1,2}^{2}-\varepsilon^{3} \frac{4}{105} h_{1,2}^{3}\right)
\end{aligned}
$$

which does not match the expression previously derived in Eq. (72)

\subsubsection{Area Equivalent Sphere}

Another interpretation of the idea is that the vaporization rate of the deformed shape should equal to the vaporization rate of a sphere with the same surface area. Comparing to the classical solution for spherical droplets, the only difference will be the definition of the droplet radius. Using Eq. (77) and the formula for sphere surface area, it can be demonstrated that the radius of the sphere with equivalent area to the droplet is

$$
r_{\text {equiv }}=1+\varepsilon^{2} \frac{1}{5} h_{1,2}^{2}-\varepsilon^{3} \frac{2}{105} h_{1,2}^{3}
$$

Substitution of this into the classical soltuion for spherical droplet vaporization yields,

$$
\dot{m}_{\text {equiv }}=-4 \pi f_{0,0}\left(1+\varepsilon^{2} \frac{1}{5} h_{1,2}^{2}-\varepsilon^{3} \frac{2}{105} h_{1,2}^{3}\right)
$$

which does not match the expression previously defined in Eq. (72)

\subsection{Curvature Effects}

These results demonstrate that the area is not the mechanism defining the increase in vaporization rate seen in these problems. Another idea is attributed to [44], that the vaporization rate of the deformed shape should relate in some way to the interface curvature. However, for non-spherical surfaces, the definition of curvature is ambiguous because any surface in three dimensional space has two principal curvatures. Therefore there are two coordinate-independent definitions of curvature. One is the mean curvature, $H$, which is half of the sum of the principal curvatures. The other is the Gaussian curvature, $K$ which is the product of the two principal curvatures. In the fluid dynamics community, the most commonly used curvature is $2 H$, due to its utility in computing the forces from surface tension. This curvature and its relationship to vaporization was tested by [35]. The Gaussian curvature is less used in fluid dynamics, however it has interesting geometrical usages. The relationship of this curvature to vaporization was explored by [31]. It may be important to note that since $K$ is the product of the principal curvatures, it has different dimensions than either the principal curvatures or $H$. Therefore a somewhat more convenient variable for analysis is $\sqrt{K}$, which is the geometric mean of the principal curvatures.

[35] studied the vaporization of droplets using numerical simulation. In this work, the droplet shape was described using a perturbation strategy similar to that used here, however, that work considers only the case where the shape perturbation consisted of a single Legendre mode. [35] extracted the local vaporization as a function of $\theta$ and compared its value to the local interface curvature. It was then discovered that the by scaling the amplitude of the curvature, an almost perfect match with the vaporization data is found. However, since this work deals with a simple perturbation of only one Legendre mode, the true implication of this work is that the vaporization rate is in phase with the curvature. It will later demonstrated that this property also holds in the current work.

[31] used an alternative approach based around their solution to the vaporizing ellipsoid. In their case, they found it easier to first compute the mean vaporization flux. Then they assert that the local flux relates to the mean flux divided by the local area, which follows from the continuity equation. After making this substitution and rearranging, they demonstrate that the local vaporization flux is

$$
F=K^{1 / 4} \frac{\dot{m}}{4 \pi}
$$

It is worth noting that Eq. 82 is exact for an ellipsoidal droplets. Accordingly, differences between Eq. (82) and the current paper are due to the representation error in assuming ellipsoidal droplets. The expression from [31] seems to be at odds with the work of [35] since it uses $K^{1 / 4}$ instead of $H$ or $K^{1 / 2}$. However, it will be shown that both expressions equivalent to order $\varepsilon$. General expressions for $H$ and $K$ are given in the Supplemental Material. Some analysis demonstrates that $H$ and $\sqrt{K}$ are equal at order $\varepsilon$, that is the (arithmetic) mean curvature and geometric mean curvature are the same to order $\varepsilon$. The expression is

$$
H=-1-\varepsilon 2 \eta_{1}
$$

where the substitution $\eta_{1}=h_{1,2} P_{2}$ was assumed.

It is clear that as $\varepsilon \rightarrow 0$, the equation recovers a curvature of unity. This is consistent with expectation. It also follows from this equation that the curvature is in phase with the shape perturbation itself (to first order). But since the local vaporization flux is also in phase with the perturbation (to first order), it follows that these quantities are in phase with each other. Therefore, this result is consistent with the result of [35]. Applying the first order solution to Eq. 82 yields,

$$
F=-f_{0,0}-\varepsilon f_{0,0} h_{1,2} P_{2}
$$

which is equivalent to the expression derived for

$$
\nabla \Phi \cdot \boldsymbol{n}
$$


when restricted to the surface $r=\eta$ and evaluated to the first order.

From both of these results we conclude that the curvature hypothesis may be a valuable tool to understand the droplet vaporization. However, we were only able to demonstrate this consistent to first order $\varepsilon$. At order $\varepsilon^{2}$, neither expression $H$ or $\sqrt{K}$ is in phase with the vaporization rate, so the results of [35] do not apply for droplets with more complex interface shapes. It can also be demonstrated that Eq. (82) does not apply when taken to order $\varepsilon^{2}$. The expressions for the relevant quantities are listed in the Supplemental Material.

\subsection{Combustion}

The previously discussed results pertained to the vaporization of the droplet. This section extends the result to study the droplet flame. The flame standoff, which measures the ratio of nominal flame diameter to droplet diameter, is a common tool used in the study of droplet combustion. However, it does not give information about the shape of the flame. For spherical droplets in quiescent flow, the flame will also be spherical. However, in the presence of convection the flame will deform around the droplet according the convective current. On earth, flame shape is dominated by natural convection due to buoyancy. Accordingly, the phenomena of dependence of flame shape upon droplet shape can only be studied in specialized experiments. Even still, images of burning non-spherical solid particles suggest that the flame shape has some dependence on the particle shape [45]. In microgravity, flame shape was explored by [46]. Their experiments required suspension of the droplet in place so that it no-longer maintained a spherical shape. They note that the flame is non-spherical, but it has greater sphericity than the droplet itself.

To discuss the flame shape, a rigorous definition of flame that is appropriate for computations must be introduced. A commonly used assumption in the community of combustion researchers is that the flame can be represented by a simple surface. That is, the region of combustion chemical reactions is considered to be restricted to a infinitely thin sheet. Under this assumption, the flame is identified by the isosurface $Z^{F}=0$. Neither [35] nor [31] explicitly address combustion in their work. However, [31] gives explicit ellipsoidal contours of the scalar field. In light of the infinitely thin flame shape assumption, it can be inferred that the flame shape predicted by their solution would also be ellipsoidal. In the current work, the isosurfaces of $Z$ are (to first order) spheroidal, the same naturally follows for the flame. However, it will be shown that this deviation from spherical shape is small, so that the flame in practice appears spherical.

In this case, since the isosurfaces are nospherical, the flame standoff will be a function of $\theta$. Denote this value as $r_{f}(\theta)$. The computation of this value is challenging. Recall,

$$
Z^{F}=Z_{0}^{F}+\varepsilon Z_{1}^{F}+\varepsilon^{2} Z_{2}^{F}
$$

Where the expression for the $Z_{k}$ functions are given previously. Exact inversion of this formula for $Z^{F}=0$ is challenging. An alternative approach may be to consider an approximation to the $Z^{F}=0$ isosurface that has error $O\left(\varepsilon^{3}\right)$. Since the error of the approximation is beyond the overall error of the solution, it is in fact just as valid as an exact inversion of the equation. The approximation may be found through the use of root finding strategies. To do this a good approximation of the standoff is needed to start the root finding algorithm. It can be demonstrated that the flame standoff solution for the spherical flame [41],

$$
r_{f, 0}=\frac{\log \left(\frac{Z_{\infty}-1}{Z_{s}-1}\right)}{\log \left(1-Z_{\infty}\right)}=\frac{\log (1+B)}{\log \left(1+v Y_{0, \infty}\right)}
$$

approximates the true value with error $O(\varepsilon)$. In this case the appropriate root finding strategy is Halley's Method yielding [47],

$$
r_{f}=r_{f, 0}-\frac{2 Z^{F} \partial_{r} Z^{F}}{2\left(\partial_{r} Z^{F}\right)^{2}-Z^{F} \partial_{r} \partial_{r} Z^{F}}
$$

A somewhat more useful quantity for analysis is the ratio $r_{f} / r_{f, 0}$, and it is

$$
\frac{r_{f}}{r_{f, 0}}=1-\varepsilon \frac{Z_{1}}{r_{f, 0} \partial_{r} Z_{0}}+\varepsilon^{2} \frac{Z_{1}^{2}+2\left(Z_{1} \partial_{r} Z_{1}-Z_{2}-\partial_{r} Z_{2}\right)}{2 r_{f, 0}\left(\partial Z_{0}^{2}\right)}
$$

Here the expression was expanded to order, and it was used that $Z_{0}\left(r_{f, 0}\right)=0$. It can be seen directly from this expression that as $r_{f, 0} \rightarrow \infty$, the ratio approaches unity. This serves as an initial explanation for why in practice flames of all droplets seem spherical. The deviation from spherical shape is inversely proportional to $r_{f, 0}$. Although [31] did not study flame shape, this is also consistent with their result. Their work suggests that contours of the scalar field are a family of confocal ellipsoids. Such a family has the property of decreasing eccentricity for increasing major axis length, which follows from the definition of eccentricity. Therefore for scalar contours that correspond to large values of major axis, the contour will be approximately spherical. Assuming the flame corresponds to such a contour, this implies a nearly spherical flame.

Another interesting note comes directly from the function $Z_{1}$ which satisfies

$$
\lim _{r \rightarrow \infty} \frac{Z_{1}}{Z_{0}}=0
$$

and a similar relation holds for $Z_{2}$. Accordingly, when viewed from sufficiently far from the droplet surface the spherically symmetric $Z_{0}$ solution dominates. Since the 
functions $Z_{1}, Z_{2}$ approach their asymptotic values rapidly, for practical values of flame standoff the solution will appear spherically symmetric. This discussion can be made stronger with further analysis of the flame surface.

Another value considered useful in combustion is the flame surface area, which can be used to make predictions about the combustion rate. It satisfies,

$$
A_{f}=2 \pi \int\left[r_{f}^{2}+\frac{1}{2}\left(\partial_{\theta} r_{f}\right)^{2}\right] \sin \theta d \theta
$$

where the expression is appropriate to order $\varepsilon^{2}$. Noting $A_{f, 0}=4 \pi r_{f, 0}^{2}$ and expanding yields,

$$
\begin{aligned}
& \frac{A_{f}}{A_{f, 0}}=1+\frac{\varepsilon^{2}}{r_{f, 0}^{2}\left(\partial_{r} Z_{0}\right)^{2}} \int \\
& {\left[\left(1+r_{f, 0}\right) Z_{1}^{2}+\frac{1}{2}\left(\partial_{\theta} Z_{1}\right)^{2}+2 r_{f, 0}\left(Z_{1} \partial_{r} Z_{1}-Z_{2} \partial_{r} Z_{0}\right)\right] \sin \theta d \theta}
\end{aligned}
$$

The solution demonstrates that the flame area does not deviate from the spherical solution at order $\varepsilon$. Accordingly, the flame is well approximated as spherical even for cases where $r_{f, 0}$ is not very large compared to the droplet radius. This result represents another mechanism why in practice flames seem to be spherical in shape. It demonstrates that the nonspherical effect is second order in $\varepsilon$.

It would be interesting to note if Eq. (92), can be used to gain trends as to the relative magnitudes of $A_{f}, A_{f, 0}$. It is clear that both $Z_{1}^{2}$ and $\left(\partial_{\theta} Z_{1}\right)^{2}$ are positive definite. However the sign of the remaining two terms is challenging to evaluate $a$ priori. However, through the computation of several numerical experiments using various boundary conditions on $Z$, it was discovered that $A_{f} \geq A_{f, 0}$ (see Section 4.5). This agrees with intuition. Since the vaporization rate is higher for the deformed droplet, the velocity of outward flowing gas is also higher, and so is the strain in the flow field. In order for the flame to experience the same level of flow strain as the spherical solution, it must there be further from the surface.

\subsection{Effects of Weber and Interface Saturation Numbers}

To explore the behavior of the model several numerical tests were performed under varying conditions. The analytical solutions are demonstrated in Section 3 , however, the solution is challenging to deal with in practice. This is because it involves the differentiation and integration of several special functions such as the Legendre and Bessel functions. To handle this a simple numerical code was written to perform these differentiations and integrations. The code is greatly simplified by the fact that the functions are separable and therefore can be written as products of pure functions of $r$ with pure functions of $\theta$. Since these separated functions depend on only one variable, standard numerical strategies can be used for their solution. The solutions were computed using standard, second order schemes from the Scientific Python environment [48,49]. From trial-and-error, it was discovered that a domain 20 diameters in length was sufficient to accurately recover the system dynamics because all functions decay rapidly as a function of the radius, i.e. $r \in[0,20]$. The full range of $\theta$ values $(\theta \in[0, \pi])$ were used. Approximately 2000 nodes were used in $r$ and 500 in $\theta$ for the studies presented here.

The target application of this work is spray combustion, and in particular the goal is in understanding the combustion of common hydrocarbon fuels in air. In such a configuration, there is only one relevant oxidizer which is the oxygen in atmospheric air, and we assume that at farfield conditions the gas is pure air. In this case the mass fraction of oxidizer at infinity is about 0.23 . It is also noted that for many hydrocarbon fuels, the value of the stoichiometric ratio is approximately $v=3.5$. Hence, for all studies a fixed value of $Z_{\infty}^{F}=-0.805$ is used. The other values of the study were varied systematically to explore their influence on the vaporization rate and the flame area ratio. Comparisons are made to the classical sphere solution. Comparisons of the vaporization rate to [31] and [35] are given in Section 4.1. Direct comparison to [31] and [35] for the flame shape are not possible, since they did not study combustion.

Figure $2 \mathrm{a}$ shows that increasing the Weber number increases the vaporization rate when compared to the base spherical droplet, as expected. The trend is independent of the interface value $Z_{s}^{F}$. Figure $2 \mathrm{~b}$ demonstrates that the flame area also appears to increase with Weber number. Further, it can be seen that the flame area ratio is significantly more sensitive to the surface saturation condition through $Z_{s}^{F}$. This is not entirely surprising. Given that the far field condition is fixed, the surface condition directly predicts the standoff of the flame. Using the arguments of Section 4.4, it is expected that the flame shape will be more sensitive to droplet deformation as it gets closer to the droplet surface. This is exactly what is demonstrated by changing $Z_{s}^{F}$ in these cases.

As noted previously, this work does not perform a true analysis of boundary layer development over the droplet or the associated convective heat and mass transfer. Therefore it may be expected that when these phenomena are accounted for, nontrivial Reynolds number effects may occur. Recent work presented by the author's group at the 2020 APS Division of Fluid Dynamics Conference [50] has explored droplet vaporization at high Reynolds number using direct numerical simulation. Data from that work suggests that even at Reynolds numbers as high as $R e=120$, the droplet vaporization rate (at fixed $R e$ ) can be represented as a polynomial function of $W e$ multiplied by the sphere solution. In this case, however, the function is found via numerical regression rather than using Eq. (72). One major difference of this result compared to Eq. (72) is that the resulting polynomial function has non-zero linear dependence on We. Detailed investigation of these high $R e$ effects will be the focus of future work. 


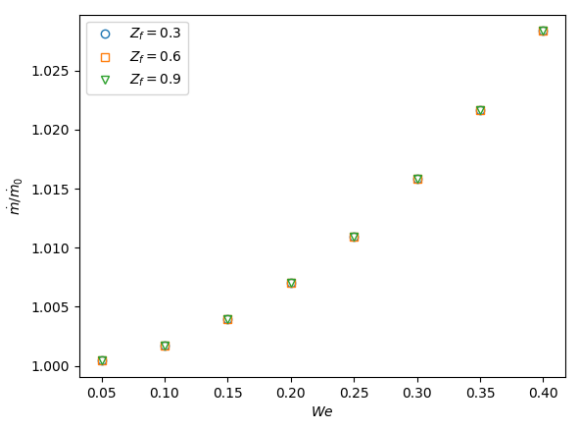

(a)

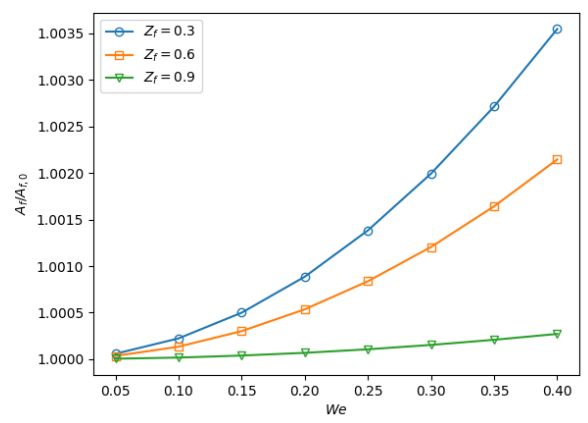

(b)

Fig. 2: Flame area ratio against Weber number. Symbols and curves indicate constant values of $Z_{s}^{F}$.

\section{Conclusion}

We have demonstrated an analytical expression for the vaporization of non-spherical droplets using the perturbation theory. For a droplet the perturbation parameter can be related to the easily measured Weber number. In particular, the work suggests that the vaporization rate is a separable, and it can be represented as a classical spherical solution multiplied with a polynomial function of Weber number. Therefore, this result can be used to refine experimental studies of droplet vaporization and combustion. The work further demonstrates that previously established results by [35. 31] create accurate first order (in Weber number) approximations of the true vaporization rates, but deviate at higher order. It is worth noting that the expression of [31] is exact for ellipsoids, so the difference between their results and current paper demonstrates the importance of representing droplet shape using a physics-based approach.

For combustion the flames demonstrate slightly nonspherical flames. However, due to three mechanisms, the flames are in practice very nearly spherical. The mechanisms are: the fact that the flame area is quadratic in $W e$ which is small, the fact that the perturbation functions decay rapidly as functions of distance from the droplet surface, and the fact that perturbation to the flame area ratio is inversely proportional to the flame standoff.

Future work will perform a more robust analysis considering boundary layer effects via a more detailed asymptotic analy- sis strategy.

\section{References}

[1] Loth, E., 2008, "Quasi-steady shape and drag of deformable bubbles and drops," International Journal of Multiphase Flow, 34(6), pp. 523-546.

[2] Liu, H., Guo, Y., and Lin, W., 2016, "Numerical simulations of spray jet in supersonic crossflows using an Eulerian approach with an SMD model," International Journal of Multiphase Flow, 82, pp. 49-64.

[3] Xu, Y., Farouk, T. I., Hicks, M. C., and Avedisian, C. T., 2020, "Initial diameter effects on combustion of unsupported equi-volume n-heptane/iso-octane mixture droplets and the transition to cool flame behavior: Experimental observations and detailed numerical modeling," Combustion and Flame, 220, pp. 82-91.

[4] Cuoci, A., Avedisian, C. T., Brunson, J. D., Guo, S., Dalili, A., Wang, Y., Mehl, M., Frassoldati, A., Seshadri, K., Dec, J. E., and Lopez-Pintor, D., 2021, "Simulating combustion of a seven-component surrogate for a gasoline/ethanol blend including soot formation and comparison with experiments," Fuel, 288(July 2020), p. 119451.

[5] van der Voort, D. D., Dam, N. J., Clercx, H. J. H., and van de Water, W., 2018, "Characterization of sprayinduced turbulence using fluorescence PIV," Experiments in Fluids, 59(7), pp. 1-7.

[6] Williams, A. N., Seitzman, J. M., and Zinn, B. T., 2020, "Visualization of autoignition kernels in jet-a fuel spray using high-speed oh plif," AIAA Propulsion and Energy Forum, pp. 1-17.

[7] Chiu, H. H., and Liu, T. M., 1977, "Group Combustion of Liquid Droplets," Combustion Science and Technology, 17(3-4), dec, pp. 127-142.

[8] Brzustowski, T. A., Twardu, E. M., Wojcicki, S., and Sobiesiak, A., 1979, "Interaction of two burning fuel droplets of arbitrary size," AIAA Journal, 17(11), pp. 1234-1242.

[9] Chiu, H. H., Kim, H. Y., and Croke, E. J., 1982, "Internal group combustion of liquid droplets," Symposium (International) on Combustion, 19(1), pp. 971-980.

[10] Law, C., Prakash, S., and Sirignano, W., 1977, "Theory of convective, transient, multicomponent droplet vaporization," Symposium (International) on Combustion, 16(1), jan, pp. 605-617.

[11] Jog, M. A., Ayyaswamy, P. S., and Cohen, I. M., 1996, "Evaporation and combustion of a slowly moving liquid fuel droplet: Higher-order theory," Journal of Fluid Mechanics, 307, pp. 135-165.

[12] Gogos, G., Sadhal, S. S., Ayyaswamy, P. S., and Sundararajan, T., 1986, "Thin-flame theory for the com- 
bustion of a moving liquid drop: Effects due to variable density," Journal of Fluid Mechanics, 171, pp. 121144.

[13] Rangel, R. H., and Fernandez-Pello, A. C., 1984 , "Mixed convective droplet combustion withl internal circulation," Combustion Science and Technology, 42(1-2), pp. 47-65.

[14] Renksizbulut, M., and Yuen, M. C., 1983, "Numerical study of droplet evaporation in a hightemperature stream," JOURNAL OF HEAT TRANSFER-TRANSACTIONS OF THE ASME, 105(2), pp. 389-397.

[15] Jackson, G. S., Avedisian, C. T., and Yang, J. C., 1992, "Observations of soot during droplet combustion at low gravity: heptane and heptane/monochloroalkane mixtures," International Journal of Heat and Mass Transfer, 35(8), pp. 2017-2033.

[16] Yao, G. F., Abdel-Khalik, S. I., and Ghiaasiaan, S. M., 2003, "An investigation of simple evaporation models used in spray simulations," JOURNAL OF HEAT TRANSFER-TRANSACTIONS OF THE ASME, 125(1), pp. 179-182.

[17] Wu, G., Sirignano, W. A., and Williams, F. A., 2011, "Simulation of transient convective burning of an noctane droplet using a four-step reduced mechanism,' Combustion and Flame, 158(6), jun, pp. 1171-1180.

[18] Alam, F. E., Liu, Y. C., Avedisian, C. T., Dryer, F. L., and Farouk, T. I., 2015, "n-Butanol droplet combustion: Numerical modeling and reduced gravity experiments," Proceedings of the Combustion Institute, 35(2), pp. 1693-1700.

[19] Liu, Y. C., Xu, Y., Avedisian, C. T., and Hicks, M. C., 2015, "The effect of support fibers on micro-convection in droplet combustion experiments," Proceedings of the Combustion Institute, 35(2), pp. 1709-1716.

[20] Sazhin, S. S., 2017, "Modelling of fuel droplet heating and evaporation: Recent results and unsolved problems," Fuel, 196, pp. 69-101.

[21] Abramzon, B., and Sirignano, W. A., 1989, "Droplet vaporization model for spray combustion calculations," International Journal of Heat and Mass Transfer, 32(9), pp. 1605-1618.

[22] Miller, R. S., and Bellan, J., 1999, "Direct numerical simulation of a confined three-dimensional gas mixing layer with one evaporating hydrocarbon-droplet-laden stream," Journal of Fluid Mechanics, 384(3), pp. 293338.

[23] Schlottke, J., and Weigand, B., 2008, "Direct numerical simulation of evaporating droplets," Journal of Computational Physics, 227(10), pp. 5215-5237.

[24] Hardt, S., and Wondra, F., 2008, "Evaporation model for interfacial flows based on a continuum-field repre- sentation of the source terms," Journal of Computational Physics, 227(11), pp. 5871-5895.

[25] Ma, C., and Bothe, D., 2013, "Numerical modeling of thermocapillary two-phase flows with evaporation using a two-scalar approach for heat transfer,' Journal of Computational Physics, 233, pp. 552-573.

[26] Rueda Villegas, L., Alis, R., Lepilliez, M., and Tanguy, S., 2016, "A Ghost Fluid/Level Set Method for boiling flows and liquid evaporation: Application to the Leidenfrost effect," Journal of Computational Physics, 316, pp. 789-813.

[27] Anumolu, L., and Trujillo, M. F., 2018, "Gradient augmented level set method for phase change simulations," Journal of Computational Physics, 353, pp. 377-406.

[28] Palmore Jr, J., and Desjardins, O., 2019, “A volume of fluid framework for interface-resolved simulations of vaporizing liquid-gas flows," Journal of Computational Physics, 399.

[29] Saufi, A. E., Frassoldati, A., Faravelli, T., and Cuoci, A., 2021, "Interface-resolved simulation of the evaporation and combustion of a fuel droplet suspended in normal gravity," Fuel, 287(August 2020), p. 119413.

[30] Grow, D. T., 1990, "Mass and Heat Transfer to an Ellipsoidal Particle," Combustion and Flame, 213, pp. 209213.

[31] Tonini, S., and Cossali, G. E., 2013, “An exact solution of the mass transport equations for spheroidal evaporating drops," International Journal of Heat and Mass Transfer, 60(1), pp. 236-240.

[32] Tonini, S., and Cossali, G. E., 2016, “One-dimensional analytical approach to modelling evaporation and heating of deformed drops," International Journal of Heat and Mass Transfer, 97, pp. 301-307.

[33] Tonini, S., and Cossali, G. E., 2018, "Modelling of heat and mass transfer from spheroidal drops with nonuniform surface temperature," International Journal of Heat and Mass Transfer, 121(2018), pp. 747-758.

[34] Taylor, T. D., and Acrivos, A., 1964, "On the deformation and drag of a falling viscous drop at low Reynolds number," Journal of Fluid Mechanics, 18(03), mar, p. 466.

[35] Mashayek, F., 2001, "Dynamics of evaporating drops. Part I: Formulation and evaporation model," International Journal of Heat and Mass Transfer, 44(8), pp. 1517-1526.

[36] Law, C. K., 1982, "Recent advances in droplet vaporization and combustion," Progress in Energy and Combustion Science, 8(3), pp. 171-201.

[37] Batchelor, G. K., 1967, An Introduction to Fluid Dyanmics, Cambridge University Press. 
[38] Haywood, R. J., Renksizbulut, M., and Raithby, G. D., 1994, "Numerical solution of deforming evaporating droplets at intermediate reynolds numbers," Numerical Heat Transfer; Part A: Applications, 26(3), pp. 253272.

[39] Helenbrook, B. T., and Edwards, C. F., 2002, "Quasisteady deformation and drag of uncontaminated liquid drops," International Journal of Multiphase Flow, 28(10), pp. 1631-1657.

[40] Korn, G. A., and Korn, T. M., 2000, Mathematical Handbook for Scientists and Engineers, Dover.

[41] Sirignano, W., 2010, Fluid Dynamics and Transport of Droplets and Spray, 2nd ed, Cambridge University Press.

[42] Joseph, D. D., 1973, "Domain perturbations: The higher order theory of infinitesimal water waves," Archive for Rational Mechanics and Analysis, 51(4), jan, pp. 295-303.

[43] NIST's Digital Library of Mathematical Functions.

[44] Lian, Z. W., and Reitz, R. D., 1993, “The effect of vaporization and gas compressibility on liquid jet atomization," Atomization and Sprays, 3.

[45] Lee, H., and Choi, S., 2018, "Volatile flame visualization of single pulverized fuel particles," Powder Technology, 333, pp. 353-363.

[46] Gokalp, I., Chauveau, C., Richard, J. R., Kramer, M., and Leuckel, W., 1989, "Observations on the low temperature vaporization and envelope or wake flame burning of n-heptane droplets at reduced gravity during parabolic flights," Symposium (International) on Combustion, 22(1), pp. 2027-2035.

[47] Press, W. H., Teukolsky, S. A., Vetterling, W. T., and Flannery, B. P., 2007, Numerical Recipes in C++., 3rd ed, Cambridge University Press, New York.

[48] Virtanen, P., Gommers, R., Oliphant, T. E., Haberland, M., Reddy, T., Cournapeau, D., Burovski, E., Peterson, P., Weckesser, W., Bright, J., van der Walt, S. J., Brett, M., Wilson, J., Millman, K. J., Mayorov, N., Nelson, A. R. J., Jones, E., Kern, R., Larson, E., Carey, C. J., Polat, İ., Feng, Y., Moore, E. W., VanderPlas, J., Laxalde, D., Perktold, J., Cimrman, R., Henriksen, I., Quintero, E. A., Harris, C. R., Archibald, A. M., Ribeiro, A. H., Pedregosa, F., van Mulbregt, P., and SciPy 1.0 Contributors, 2020, "SciPy 1.0: Fundamental Algorithms for Scientific Computing in Python," Nature Methods, 17, pp. 261-272.

[49] Harris, C. R., Millman, K. J., van der Walt, S. J., Gommers, R., Virtanen, P., Cournapeau, D., Wieser, E., Taylor, J., Berg, S., Smith, N. J., Kern, R., Picus, M., Hoyer, S., van Kerkwijk, M. H., Brett, M., Haldane, A., Fernández del Río, J., Wiebe, M., Peterson, P., Gérard-Marchant, P., Sheppard, K., Reddy, T.,
Weckesser, W., Abbasi, H., Gohlke, C., and Oliphant, T. E., 2020, "Array programming with NumPy," $\mathrm{Na}$ ture, 585, p. 357-362.

[50] Setiya, M., and Palmore Jr, J., 2020, "Effect of straining flow and droplet shape on vaporization rate of liquid fuel droplet," At 73rd Annual Meeting of the APS Division of Fluid Dynamics, American Physical Society. 